\title{
Westerlund 1 is a Galactic Treasure Chest: The Wolf-Rayet Stars
}

\author{
C. K. Rosslowe and P. A. Crowther \\ Department of Physics and Astronomy, University of Sheffield \\ email: chris.rosslowe@shef.ac.uk
}

\begin{abstract}
The Westerlund 1 Galactic cluster hosts an eclectic mix of coeval massive stars. At a modest distance of $4-5 \mathrm{kpc}$, it offers a unique opportunity to study the resolved stellar content of a young $(\sim 5 \mathrm{Myr})$ high mass $\left(5 \cdot 10^{4} M_{\odot}\right)$ star cluster. With the aim of testing single-star evolutionary predictions, and revealing any signatures of binary evolution, we discuss on-going analyses of NTT/SOFI near-IR spectroscopy of Wolf-Rayet stars in Westerlund 1. We find that late WN stars are H-poor compared to their counterparts in the Milky Way field, and nearly all are less luminous than predicted by single-star Geneva isochrones at the age of Westerlund 1.
\end{abstract}

Keywords. stars: early-type, mass loss, Wolf-Rayet - infrared: stars - galaxies: star clusters

\section{Introduction}

Westerlund 1 (Wd1) is amongst the most massive young clusters in the Galaxy. We witness this coeval collection of stars at an interesting epoch, as Clark et al. (2005) have identified a plethora of post-main sequence massive stars, indicating an age of $5 \pm 1 \mathrm{Myr}$ and a main sequence turn-off at approximately $30-35 M_{\odot}(\mathrm{O} 7 \mathrm{~V})$.

A high fraction of dust producing WC stars and coincidental hard X-ray sources amongst the observed Wolf-Rayet (WR) stars suggests a binary fraction approaching unity (Crowther et al. 2006). Indeed, Schneider et al. (2014) predict that after only a few Myr, the majority of a cluster's most luminous stars are the products of binary interaction. Here we report on preliminary tailored spectral analyses of $15 \mathrm{WR}$ stars in Wd1 from Crowther et al. (2006), and discuss how derived parameters compare to single-star and binary evolutionary models.

\section{Data \& Analysis Method}

We obtained NTT/SOFI spectra of $23 \mathrm{WR}$ stars in Wd1 using IJ and HK grisms ( $\mathrm{R} \sim 1000$ ), identified by differential narrow-band imaging (Crowther et al. 2006).

We have carried out spectral modelling of 15 of these WR stars (neglecting very late WN9-10 or dusty WC) - 2 of which are shown in Fig 1 - using the CMFGEN model atmosphere code (Hillier \& Miller 1998) to derive effective temperatures, luminosities, abundances, mass-loss rates and wind velocities.

We constrain luminosities and extinction simultaneously, by requiring model spectral energy distributions to match a combination multi-band photometry and flux calibrated spectra, as shown in Fig. 2.

\section{Results}

Most WR stars are less luminous than single-star Geneva isochrones covering the expected age of Westerlund 1; older ages are precluded by the presence of high-mass main sequence stars. Late WN stars are generally H-poor compared to their field Milky 

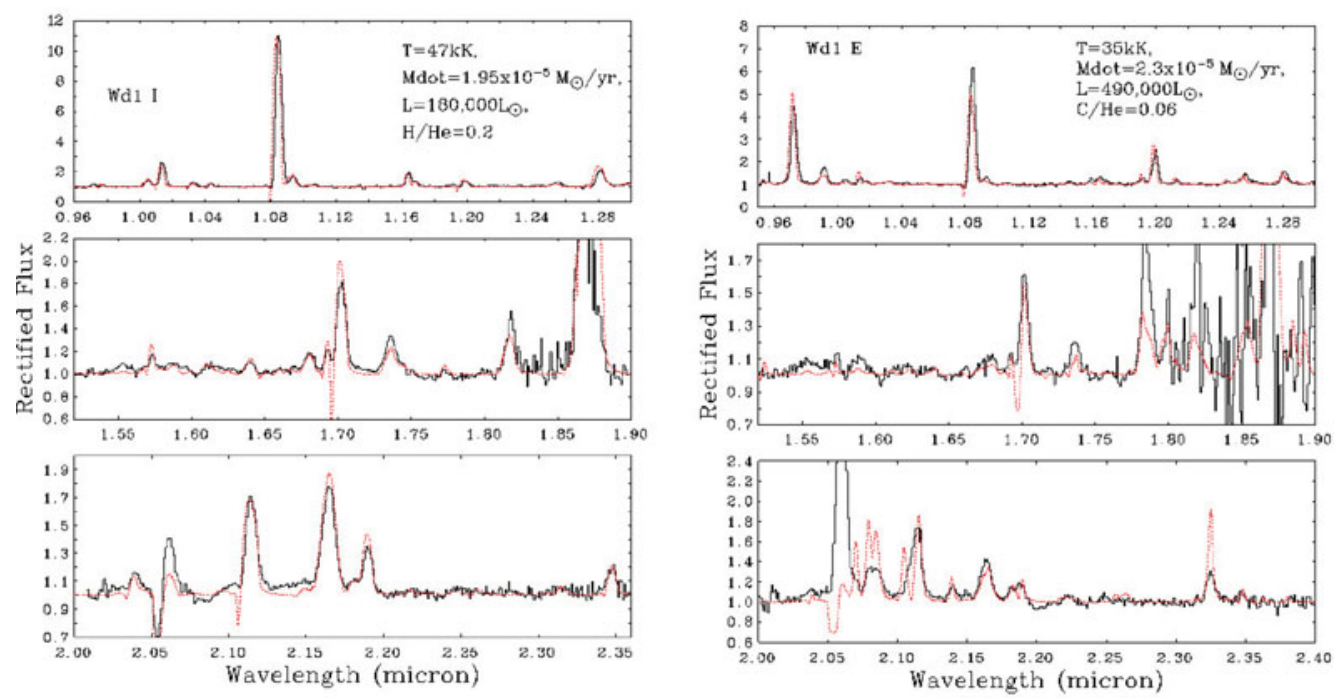

Figure 1. Left. NTT/SOFI spectrum (solid) and corresponding CMFGEN model (dotted) for Wd1-I (WN8). Right As left for Wd1-E (WC9). CMFGEN parameters are displayed in the upper panels.
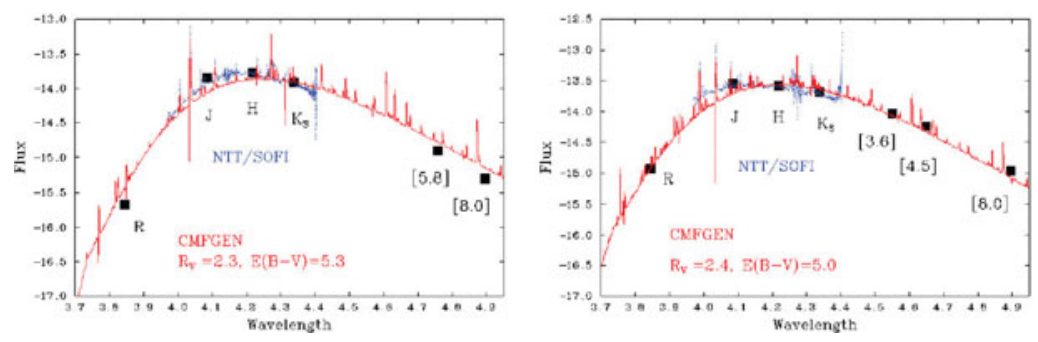

Figure 2. Left SED of Wd1-I, with flux calibrated NTT/SOFI spectra and multiband photometry (R:VLT/FORS2, JHK:SOFI, [5.8]-[8.0]:GLIMPSE). Right As left for Wd1-E. Model spectra are reddened using a Howarth (1983) extinctin law.

way counterparts. Low luminosity and $\mathrm{H}$ deficiency amongst the WN stars is consistent with outcomes of binary evolution (e.g. Eldridge et al. 2008). Preliminary mass-loss rates of the H-free early WN stars are systematically lower than those adopted by stellar models for their luminosities. Overall, The WR stars in Westerlund 1 analysed to date display properties that are inconsistent with the current generation of single-star models (Ekström et al. 2012). Analysis of the dust producing WC stars is forthcoming.

\section{References}

Clark, J. S., Negueruela, I., Crowther, P. A., \& Goodwin, S. P. 2005, A\&\&A 434, 949

Crowther, P. A., Hadfield, L. J., Clark, J. S., Negueruela, I., \& Vacca, W. D. 2006, MNRAS 372,1407

Ekström, S., Georgy, C., Eggenberger, P., et al. 2012, A\&A 537, A146

Eldridge, J. J., Izzard, R. G., \& Tout, C. A. 2008, MNRAS 384, 1109

Hillier, D. J. \& Miller, D. L. 1998, ApJ 496, 407

Howarth, I. D. 1983, MNRAS 203, 301

Schneider, F. R. N., Izzard, R. G., de Mink, S. E., et al. 2014, ApJ 780, 117 